\title{
PIFI Affects the Proliferation and Apoptosis of Cervical Cancer Cells by Influencing TERT
}

This article was published in the following Dove Press journal:

Cancer Management and Research

\author{
Jiancai Wang ${ }^{\mathrm{l}, *}$ \\ Xiaoyan Zhu',* \\ Pian Ying ${ }^{2}$ \\ Yingping $\mathrm{Zhu}^{2}$
}

'Department of Gynaecology and Obstetrics, Jianhu Hospital Affiliated to Nantong University, Yancheng, Jiangsu 224700, People's Republic of China; ${ }^{2}$ Department of Obstetrics and Gynecology, The First Affiliated Hospital of Zhejiang University of Traditional Chinese Medicine, Hangzhou, Zhejiang 310006, People's Republic of China

*These authors contributed equally to this work
Correspondence: Yingping Zhu Email ypzhupp@I26.com
Introduction: Cervical cancer is a common malignancy in female and it is a serious disease threatening women's lives. We aimed to explore whether PIF1 helicase expression could affect cell proliferation and apoptosis, and whether its mechanisms were related to the expression and activity of TERT.

Methods: Western blot analysis was used to detect the expressions of PIF1 and TERT in End1/E6E7, Hela, SiHa, Ca-Ski and C-33A cells and apoptosis-related proteins (Bax, Bcl-2 and Caspase-3). RT-qPCR and Western blot analysis determined the expressions of PIF1 and TERT after transfection. After transfection or cycloastragenol (CAG) treatment, the proliferation, apoptosis, cell cycle and telomerase TERT activity were analyzed by CCK- 8 assay, flow cytometry analysis and ELISA assay. Co-immunoprecipitation assay was used to verify the interactions between PIF1 and TERT.

Results: The expressions of PIF1 and TERT in End1/E6E7, Hela, SiHa, Ca-Ski and C-33A cells were increased. As PIF1 and TERT expressions in C-33A cells showed the minimum increase, C-33A cells were chosen for the next study. PIF1 interference inhibited the proliferation, decreased the ratio of $\mathrm{G} 2 / \mathrm{M}$ phase and promoted apoptosis of transfected cells, and PIF1 interference promoted the expressions of Bax and Caspase-3 and suppressed the Bcl-2 expression. Furthermore, PIF1 interference down-regulated the telomerase activity. The effect of PIF1 overexpression was opposite to that of PIF1 interference. Coimmunoprecipitation assay demonstrated that PIF1 could combine with TERT. CAG treatment effectively reversed the effect of PIF1 interference on proliferation, cycle and apoptosis of C-33A cells transfected with shRNA-PIF1. Moreover, CAG treatment increased the expressions of PIF1 and TERT.

Discussion: PIF1 helicase could promote the proliferation and suppress the apoptosis of cervical cancer cells by down-regulating the activity of telomerase TERT.

Keywords: PIF1, proliferation, apoptosis, cervical cancer cell, TERT

\section{Introduction}

In the world, cervical cancer is the fourth most common malignancy in women, following breast cancer, colorectal cancer and lung cancer. ${ }^{1}$ Every year, more than half a million women worldwide are diagnosed with cervical cancer and there are more than 300,000 deaths from cervical cancer. ${ }^{2}$ According to the latest National Cancer statistics released by the National Central Cancer of China (NCCC) in 2017, cervical cancer accounted for $6.25 \%$ of all cancers, ranking sixth among the top 10 female malignant tumors and cervical cancer death accounted for about $3.96 \%$, ranking eighth among the top 10 malignant tumors of deaths. Therefore, identifying effective therapeutic methods is currently urgent in cervical cancer research. 
Telomerase plays a key role in regulating cell proliferation and is present in most of the infinite proliferation processes of tumor cells. ${ }^{3}$ Telomerase TERT induced the proliferation of tumor cells, and the expression of telomerase TERT was upregulated in tumor tissues, but it was not expressed or low expressed in normal tissues. ${ }^{4}$ Some researchers considered that the up-regulation of TERT mRNA would lead to an increase of telomerase activity, which was essential for cell proliferation, differentiation, aging and the occurrence and development of tumors. ${ }^{5}$ A study has shown that telomerase TERT expression was relatively high in cervical cancer, and increased expression of telomerase TERT could promote the tumor development. ${ }^{6,7}$ The inhibition of TERT gene expression or enzyme activity could suppress the tumor growth. ${ }^{8}$

PIF1 helicase has an effect on telomerase activity. ${ }^{9}$ PIF1 helicase could inhibit TERT activity and dissociate telomeres, thus promoting cell apoptosis. ${ }^{10}$ PIF1 helicase directly dissociated the telomerase complex from the telomerase DNA terminal to inhibit the telomerase lengthening telomeres. ${ }^{10}$ In the process of tumor growth, human PIF1 helicase could promote the activity of TERT protein, but had no direct effect on its mRNA level. PIF1 helicase was shown to promote DNA replication and tumor cell proliferation. ${ }^{11,12}$ The TCGA database showed that the expression level of PIF1 helicase was significantly increased in cervical cancer, but there was no direct reports about it. In addition, whether PIF1 can affect the proliferation and invasion of tumor cells by affecting TERT expression remains to be discussed.

Therefore, this study was to explore the expression of PIF1 helicase in cervical cancer cells and whether its expression level affected cell proliferation and apoptosis, and whether its mechanisms were related to the TERT expression.

\section{Materials and Methods Cell Culture}

End1/E6E7, Hela and SiHa cells were obtained from American Type Culture Collection (Rockville, MD, USA). Ca-Ski and C-33A cells were brought from Shanghai lianmai biological engineering Co., Ltd. Cells were cultured in RPMI1640 culture medium containing $10 \%$ FBS, $1 \%$ penicillin-streptomycin double antibiotic. The solution was changed every 2-3 days. All cells were placed in a $37^{\circ} \mathrm{C}$ and $5 \% \mathrm{CO}_{2}$ incubator for incubation.

\section{TUNEL Assay}

The slides of C-33A cells were digested by protease $\mathrm{K}$, followed by the treatment of TdT and Biotin-dUTP. Then, slides of C-33A cells were sealed, treated with streptavidin-HRP working liquid and stained with $\mathrm{DAB}$ reagent. The apoptosis of C-33A cells was observed under the light microscope. The TUNEL assay was performed by TUNEL detection kit.

\section{Western Blot Analysis}

After the extraction of proteins in the cell, protein concentration was detected according to BCA kit. The extracted proteins were added to the buffer, which was heated to $95^{\circ} \mathrm{C}$ for $10 \mathrm{~min}$. Each well was loaded with $30 \mu \mathrm{g}$ proteins which were separated by $10 \%$ SDS-PAGE at $80-120 \mathrm{~V}$. The voltage of wet transfer and transmembrane was controlled at $100 \mathrm{mV}$, lasting 45-70 min. After proteins were transferred to PVDF membrane, the membrane was sealed in $5 \%$ skim milk powder for 1 $\mathrm{h}$ and incubated with primary antibodies [anti-PIF1 (ab192369; Abcam), anti-TERT (ab191523; Abcam), antiBax (ab32503; Abcam), anti-Bcl-2 (ab32124; Abcam), anticaspase-3 (ab2302; Abcam)] at $4^{\circ} \mathrm{C}$ overnight. After washing with buffer, the membrane was then incubated with rabbit horseradish peroxidase-linked IgG at room temperature for 1 h. After the washing by buffer for three times, the membrane was treated with chemiluminescent reagent to develop protein bands. With GAPDH as internal reference, Bio-rad Gel DolEZ imager was used for protein imaging, and Image J software was used to analyze the gray level of the target band.

\section{Cell Transfection}

C-33A cells in logarithmic phase were selected. After trypsin digestion, $2 \mathrm{~mL}$ of cell suspension was inoculated into a 6-well plate $\left(1 \times 10^{5}\right.$ cells $\left./ \mathrm{mL}\right)$ and incubated in an incubator with $5 \%$ $\mathrm{CO}_{2}$ at $37^{\circ} \mathrm{C}$ for $24 \mathrm{~h}$. C-33A cells were respectively transfected with shRNA-PIF1-1, shRNA-PIF1-2, OverExp-PIF1-1 and OverExp-PIF1-2 for $48 \mathrm{~h}$ according to Lipofectamine ${ }^{\mathrm{TM}} 2000$ transfection reagent description method. The transfection effect of the cells was determined by RT-qPCR.

\section{CCK-8 Assay}

C-33A cells were inoculated in 96-well plates (with cell density $1 \times 10^{4} /$ hole), each hole containing $100 \mu \mathrm{L}$ medium. After cell transfection or cycloastragenol (CAG) treatment, $10 \mu \mathrm{L}$ CCK8 solution was added to each well 1 $\mathrm{h}$ before the test, and the culture plate was incubated in the incubator for $4 \mathrm{~h}$. The optical density (OD) value at 450 $\mathrm{nm}$ was determined by an enzyme micro-plate reader. 


\section{Flow Cytometry Analysis}

Cells in each group were added with $2.5 \mathrm{~g} / \mathrm{L}$ trypsin for full digestion, and then collected and washed with precooled cold acid buffer. The cell density of cell suspension was adjusted to $10^{9} / \mathrm{L}$. A test tube was subsequently added with $100 \mu \mathrm{L}$ cell suspension, $5 \mu \mathrm{L}$ Annexin V and $10 \mu \mathrm{L}$ PI for 15 min incubation in the dark. The apoptosis rate of cells in each group was detected by flow cytometry.

\section{RT-qPCR Analysis}

TRIzol reagent was used to extract $100 \mathrm{ng}$ total RNA for reverse transcription, and the expression of the target gene was detected by SYBR Green method, with GAPDH as the control. The PCR conditions were: pre-denaturation at $95^{\circ} \mathrm{C}$ for $30 \mathrm{~s}$; PCR reaction at $95^{\circ} \mathrm{C}$ for $5 \mathrm{~s}$ and $60^{\circ} \mathrm{C}$ for $30 \mathrm{~s}, 40$ cycles. The relative gene expression was calculated by $2^{-\Delta \Delta \mathrm{Ct}}$ method.

\section{ELISA Assay}

The TERT expression in C-33A cells after transfection or CAG treatment was detected according to the ELISA Kits (NanJing JianCheng Bioengineering Institute, China). A Model 680 Microplate Reader was applied to obtain the OD value at $450 \mathrm{~nm}$.

\section{Co-Immunoprecipitation Assay}

C-33A cells in logarithmic phase were selected and lysed to prepare the samples. First, the samples were first passed through uncoated beads to absorb proteins that were not specifically bound to the beads. Second, an antibody against the target protein is added to the samples to combine with the target protein for the formation of antigen-antibody complexes. Third, the precipitated compounds were cleaned to enrich antigen-antibody complexes by removing some nonspecific binding of proteins. Finally, Western blot analysis was used to detect the expression of PIF1 and TERT.

\section{Statistical Analysis}

The experiment was repeated three times. Data processing was conducted by SPSS 17. 0 statistical software. Data were expressed as mean \pm standard deviation (SD). Student's $t$-test and one-way ANOVA single factor analysis of variance were carried out for statistical analysis. $\mathrm{P}<0.05$ showed a statistical meaning.

\section{Results \\ The Expressions of PIFI and TERT in Cervical Cancer Cells}

As shown in Figure 1, the expressions of PIF1 and TERT in cervical cancer cells were all increased compared with those in Ect1/E6E7 cells. The expressions of PIF1 and TERT in C-33A cells exhibited the minimum increase compared with other cancer cells, and therefore, C-33A cells were chosen for the subsequent experiments.

\section{The Change of PIFI Expression in Cervical Cancer Cells After Transfection}

C-33A cells were transfected with blank vector, shRNA-PIF1 $-1 / 2$ and OverExp-PIF1-1/2. The results showed that the PIF1 expression in C-33A cells transfected with shRNA-PIF1-1/2 was down-regulated and the PIF1 expression in C-33A cells transfected with OverExp-PIF1-1/2 was up-regulated. The decrease of PIF1 expression in shRNA-PIF1-1 group and increase of PIF1 expression in OverExp-PIF1-1 group are more significant than that in shRNA-PIF1-2 group and
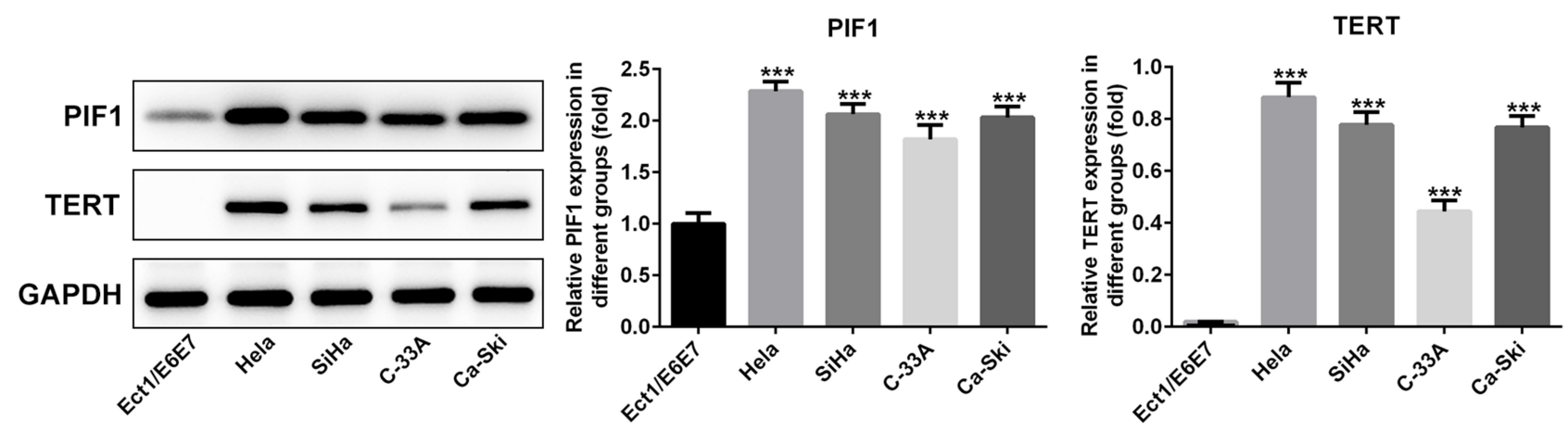

Figure I The expressions of PIFI and TERT in cervical cancer cells. The expressions of PIFI and TERT in cervical cancer cells were detected by Western blot analysis. $* * * P<0.001$ vs Ectl/E6E7 group. 


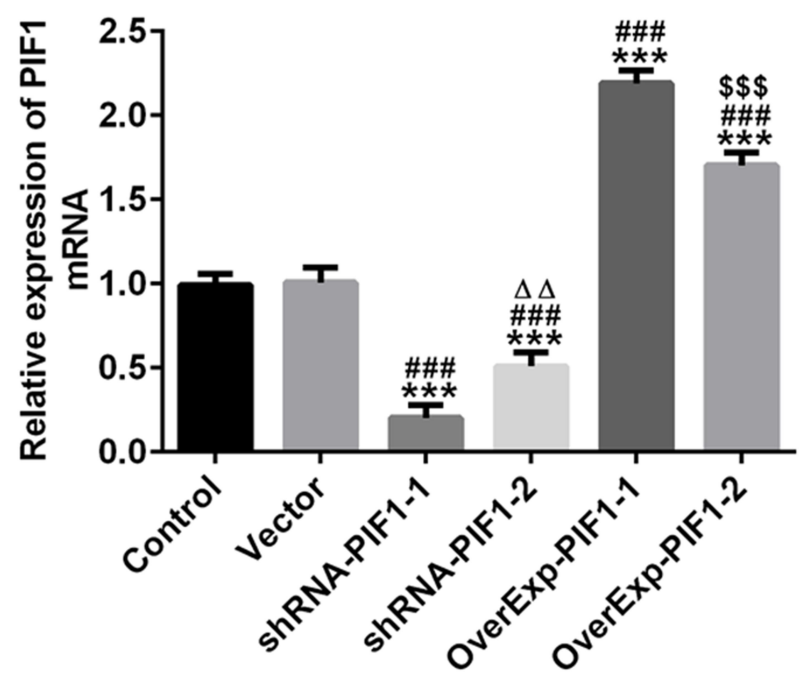

Figure 2 The change of PIFI expression in cervical cancer cells after transfection. The transfection effect was determined by RT-qPCR analysis. ***P $<0.001$ vs Control group. ${ }^{\#} \mathrm{P}<0.00$ I vs Vector group. ${ }^{\Delta \triangle} \mathrm{P}<0.0$ I vs shRNA-PIFI-I group. ${ }^{\$ \$ \$} \mathrm{P}<0.00$ I vs OverExp-PIFI-I group.

OverExp-PIF1-2 group (Figure 2). Therefore, shRNA-PIF1-1 and OverExp-PIF1-1 were selected for the subsequent experiments.

\section{The Change of PIFI Expression Affects the Proliferation, Apoptosis and Cycle of Cervical Cancer Cells}

After cell transfection, PIF1 interference inhibited the proliferation, decreased the rate of $\mathrm{G} 2 / \mathrm{M}$ phase and promoted the apoptosis of C-33A cells while PIF1 overexpression could reverse the effects of PIF1 interference on proliferation, cell cycle and apoptosis (Figure 3).

The Change of PIFI Expression Affects the mRNA and Protein Expressions of PIFI and TERT in Cervical Cancer Cells After Transfection

It has been confirmed that human PIF1 can promote TERT expression in tumor, but it has no direct effect on its mRNA level. As shown in Figure 4A and B, the mRNA and protein expressions of PIF1 were downregulated in shRNA-PIF1 transfected C-33A cells and up-regulated in OverExp-PIF1 transfected C-33A cells. As shown in Figure $4 \mathrm{C}$ and D, PIF1 interference and PIF1 overexpression respectively inhibited and promoted the TERT mRNA and protein expression. PIF1 had more obvious effect on TERT protein expression than TERT mRNA expression.

\section{The Change of PIFI Expression Affects the Expressions of Apoptosis-Related Proteins and Telomerase TERT}

Consistent with the effects of PIF1 interference and PIF1 overexpression on cell apoptosis, the expressions of Bax and Caspase-3 were enhanced and Bcl-2 expression was decreased in shRNA-PIF1 transfected C-33A cells, while the expressions of apoptosis-related proteins were reversed in OverExp-PIF1 transfected C-33A cells

A

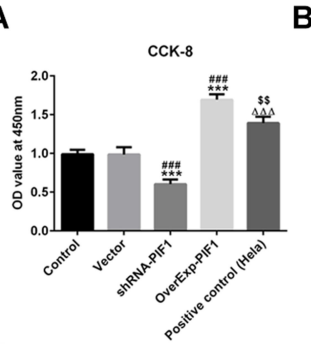

B
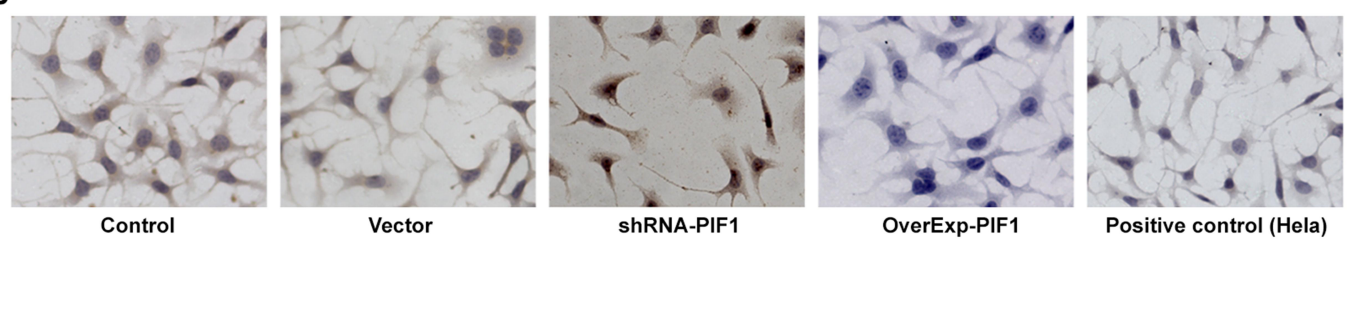

C
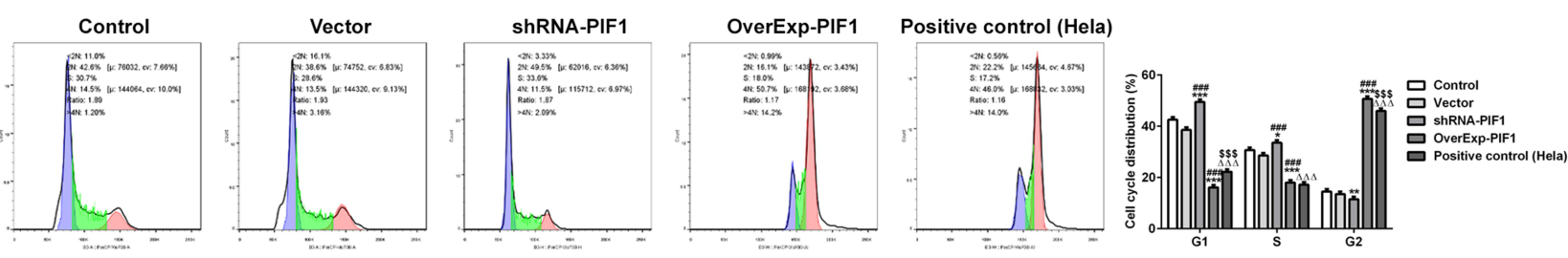

Figure 3 The change of PIFI expression affects the proliferation, apoptosis and cycle of cervical cancer cells. (A) The proliferation of C-33A cells after transfection was detected by CCK-8 assay. ${ }^{* * * P}<0.00$ I vs Control group. ${ }^{\# \# P<0.00 I}$ vs Vector group. ${ }^{\Delta \triangle \Delta} \mathrm{P}<0.001$ vs shRNA-PIFI group. ${ }^{\$ \$} \mathrm{P}<0.0$ I vs OverExP-PIFI group. (B) The apoptosis of $\mathrm{C}-33 \mathrm{~A}$ cells after transfection was detected by Tunel assay. (C) The cycle of $\mathrm{C}-33 \mathrm{~A}$ cells after transfection was detected by flow cytometry analysis. $* \mathrm{P}<0.05$, $* * \mathrm{P}<0.0 \mathrm{I}$ and ***P $<0.00$ I vs Control group. ${ }^{\# \# P} \mathrm{P}<0.00$ I vs Vector group. ${ }^{\Delta \Delta \Delta} \mathrm{P}<0.001$ vs shRNA-PIFI group. ${ }^{\$ \$} \mathrm{P}<0.00 \mathrm{I}$ vs OverExp-PIFI group. 
A

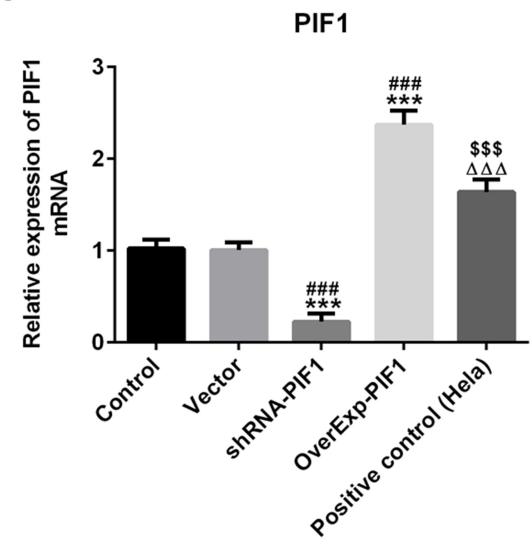

C

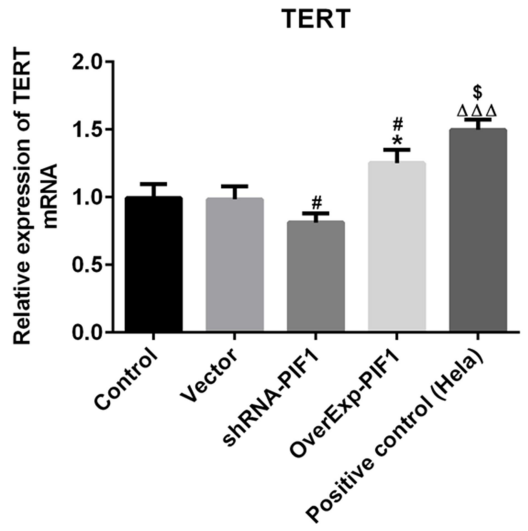

B

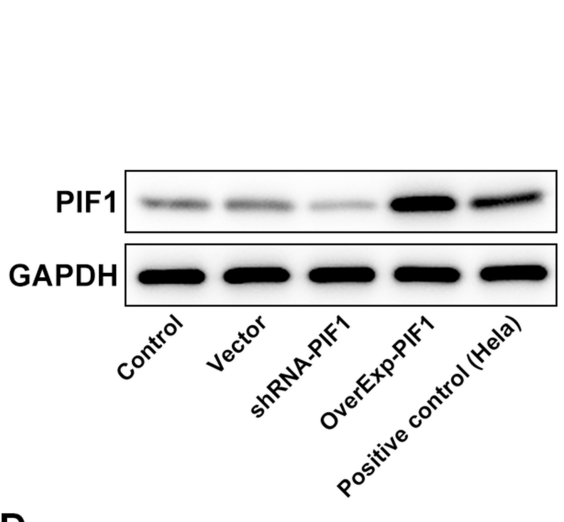

D

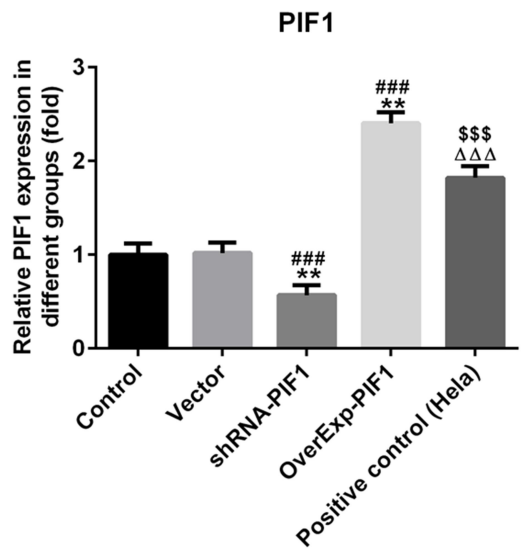

TERT

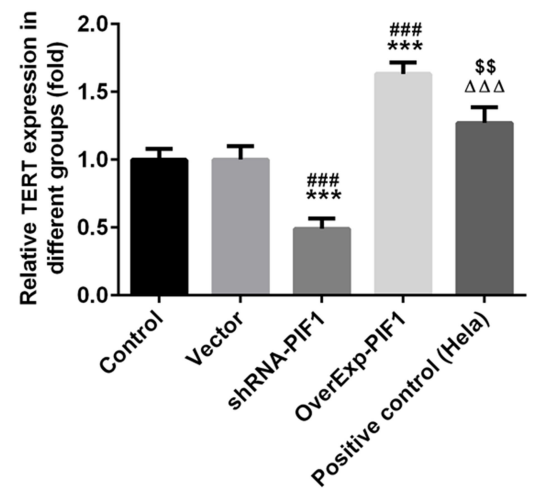

Figure 4 The change of PIFI expression affects the mRNA and protein expressions of PIFI and TERT in cervical cancer cells after transfection. (A) The PIFI

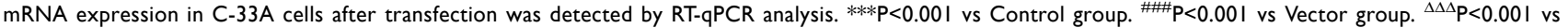
shRNA-PIFI group. ${ }^{\$ \$} \mathrm{P}<0.001$ vs OverExp-PIFI group. (B) The PIFI protein expression in C-33A cells after transfection was detected by Western blot analysis.

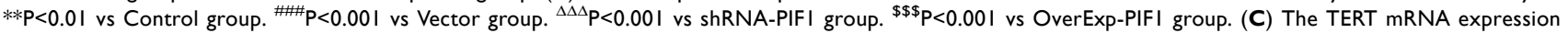
in $\mathrm{C}-33 \mathrm{~A}$ cells after transfection was detected by $\mathrm{RT}-\mathrm{qPCR}$ analysis. ${ }^{*} \mathrm{P}<0.05$ vs Control group. ${ }^{\#} \mathrm{P}<0.05$ vs Vector group. ${ }^{\Delta \Delta} \mathrm{P}<0.00 \mathrm{I}$ vs shRNA-PIFI group. ${ }^{\$} \mathrm{P}<0.05$ vs OverExp-PIFI group. (D) The TERT protein expression in C-33A cells after transfection was detected by Western blot analysis. $* * * P<0.00 \mathrm{I}$ vs Control group. ${ }^{\# \#} \mathrm{P}<0.001$ vs Vector group. ${ }^{{ }^{\Delta} \Delta} \mathrm{P}<0.001$ vs shRNA-PIFI group. ${ }^{\$ \$} \mathrm{P}<0.01$ vs OverExp-PIFI group.

(Figure 5A). As shown in Figure 5B, PIF1 interference obviously reduced the expression of telomerase TERT and PIF1 overexpression remarkably improved the expression of telomerase TERT.

\section{PIFI Can Be Combined with TERT}

After the sample was passed through uncoated beads to remove the nonspecific binding proteins, anti-TERT or anti-PIF1 was separately added to two samples to combine with the target protein for the formation of antigen-antibody complexes. As shown in Figure 6, PIF1 protein existed in anti-TERT group and TERT protein existed in anti-PIF1 group. Therefore, PIF1 could be combined with TERT.

\section{Cycloastragenol (CAG) Affects the Proliferation, Apoptosis and Cycle of Cervical Cancer Cells}

Cycloastragenol (CAG) can activate telomerase. As shown in Figure 7, CAG treatment improved the proliferation and the rate of $\mathrm{G} 2 / \mathrm{M}$ phase. What's more, it suppressed the apoptosis of C-33A cells transfected with shRNA-PIF1.

\section{Cycloastragenol (CAG) Affects the Expressions of Apoptosis-Related Proteins, PIFI and TERT}

As shown in Figure 8A, CAG treatment up-regulated the expressions of Bcl-2, PIF1 and TERT and down-regulated the expressions of Bax and Caspase- 3 in C-33A cells 
A

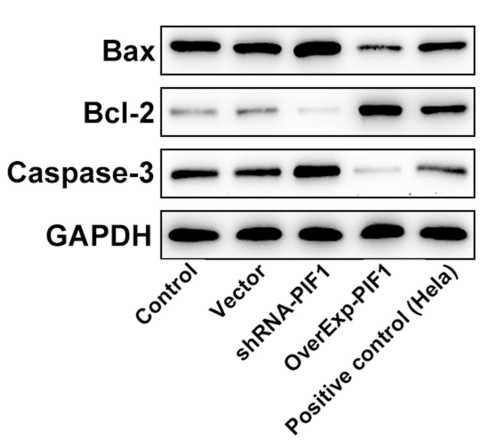

$\mathrm{Bcl}-2$

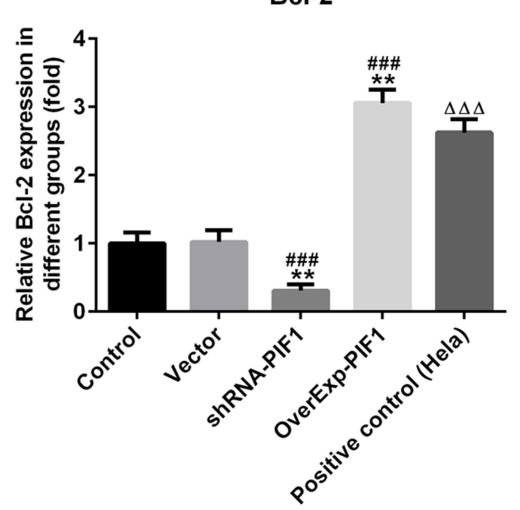

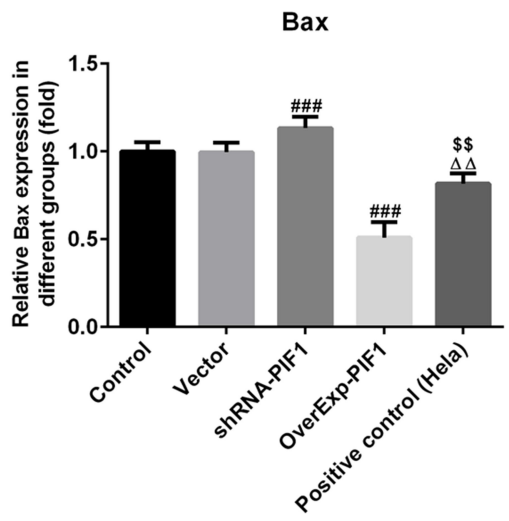

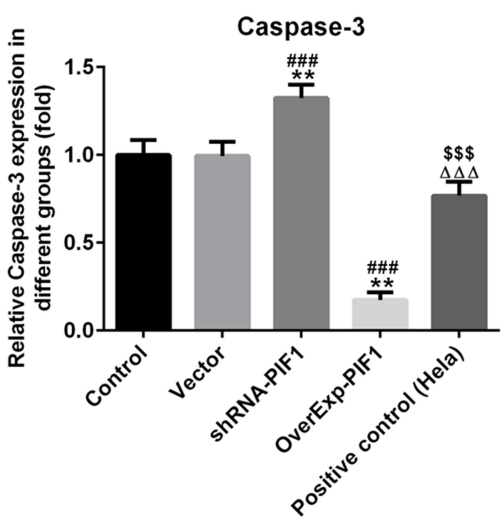

B

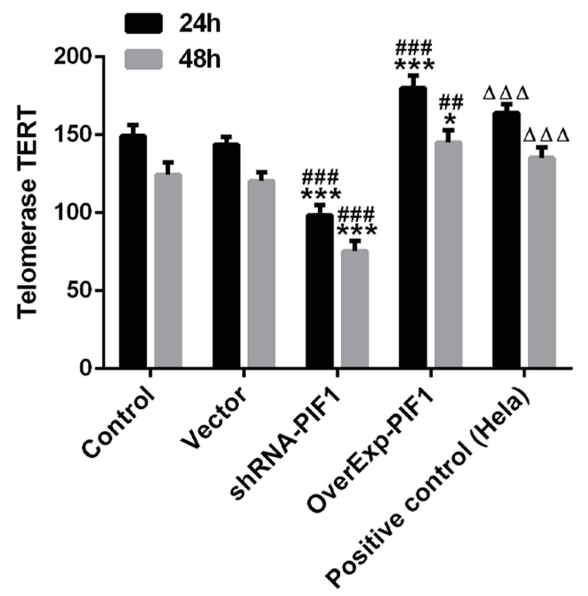

Figure 5 The change of PIFI expression affects the expressions of apoptosis-related proteins and telomerase TERT. (A) The expression of Bax, Bcl-2 and Caspase-3 in $\mathrm{C}-33 \mathrm{~A}$ cells after transfection was analyzed by Western blot analysis. ${ }^{* *} \mathrm{P}<0.0 \mathrm{I}$ vs Control group. ${ }^{\ldots} \mathrm{P}<0.00 \mathrm{I}$ vs Vector group. ${ }^{\Delta \Delta} \mathrm{P}<0.0 \mathrm{I}$ and ${ }^{\Delta \Lambda \Delta} \mathrm{P}<0.00 \mathrm{I}$ vs shRNA-PIFI group. ${ }^{\$ \$} \mathrm{P}<0.01$ and ${ }^{\$ \$ \$} \mathrm{P}<0.001$ vs OverExP-PIFI group. (B) The expression level of TERT in C-33A cells after transfection was analyzed by ELISA assay. ${ }^{* P}<0.05$ and ${ }^{* * *} \mathrm{P}<0.00 \mathrm{I}$ vs Control group. ${ }^{\# \#} \mathrm{P}<0.0 \mathrm{I}$ and ${ }^{\# \#} \mathrm{P}<0.001$ vs Vector group. ${ }^{\Delta \Delta \Delta} \mathrm{P}<0.00 \mathrm{I}$ vs shRNA-PIFI group.

transfected with shRNA-PIF1. Furthermore, CAG treatment increased the expression of telomerase TERT in C-33A cells transfected with shRNA-PIF1 (Figure 8B).

\section{Discussion}

Here, this study was to investigate whether PIF1 expression could affect the cell proliferation and apoptosis by regulating the expression of TERT. The results indicated that PIF1 helicase could promote the proliferation, increase the rate of $\mathrm{G} 2 / \mathrm{M}$ phase and suppress the apoptosis of cervical cancer cells by up-regulating the expression of telomerase TERT.
A

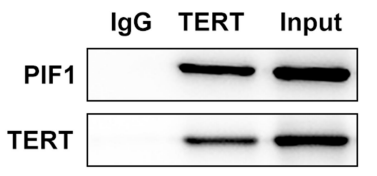

B

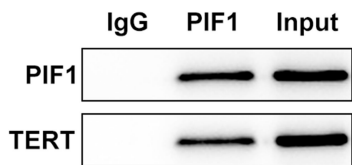

Figure 6 PIFI can be combined with TERT. The relation between PIFI and TERT was determined by Co-immunoprecipitation assay.
Telomerase is of great significance in the occurrence and development of malignant tumor. Telomerase RNA and TRET protein are the two main ingredients of telomerase. Clinical data showed that telomerase in most normal tissues and benign lesions exhibited low or undetectable level, but more than $90 \%$ of the telomerase activity in malignant tumor was high. ${ }^{13}$ All malignant tumor cells with telomerase activity had the expression of TERT. ${ }^{14}$ TERT can specifically regulate the activity of telomerase and become a rate-limiting enzyme affecting the activity of telomerase. ${ }^{15}$ There is a positive correlation between TERT and telomerase, which can greatly improve the activity of human telomerase and prolong the life of cells, thus directly leading to the transformation of normal cells into malignant cells. ${ }^{16}$ In this study, TERT expression was indeed up-regulated in cervical cancer cells.

TERT may be a good target for specific tumor suppression. ${ }^{17}$ Zhu et $\mathrm{al}^{18}$ found that HPV16E6 silencing could affect TERT to participate in the biological behaviors of human cervical cancer cells in vitro and TERT 
A

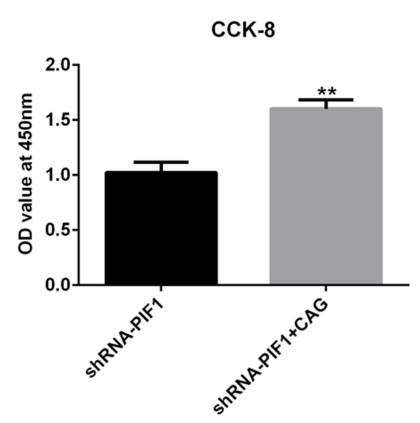

B

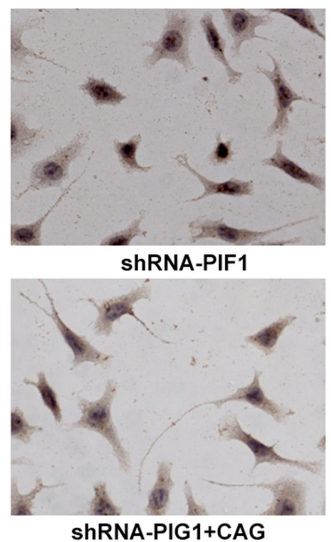

C

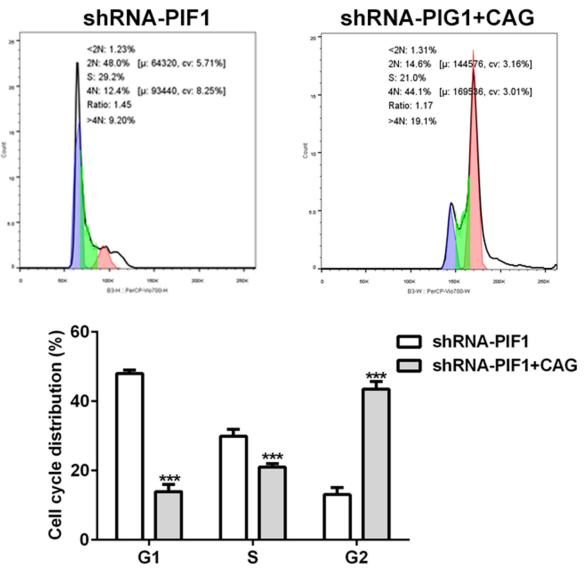

Figure 7 Cycloastragenol (CAG) affects the proliferation, apoptosis and cycle of cervical cancer cells. (A) The proliferation of C-33A cells after CAG treatment was detected by CCK-8 assay. ${ }^{* * P}<0.0$ I vs shRNA-PIFI group. (B) The apoptosis of C-33A cells after CAG treatment was detected by Tunel assay. (C) The cycle of C-33A cells after CAG treatment was detected by flow cytometry analysis. $* * * \mathrm{P}<0.001$ vs shRNA-PIFI group.

A
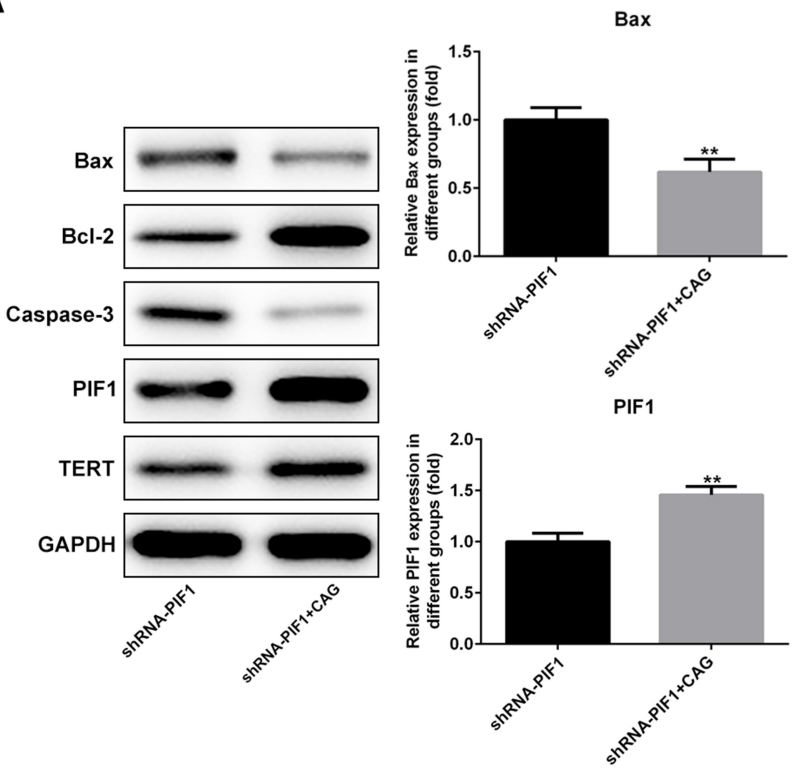

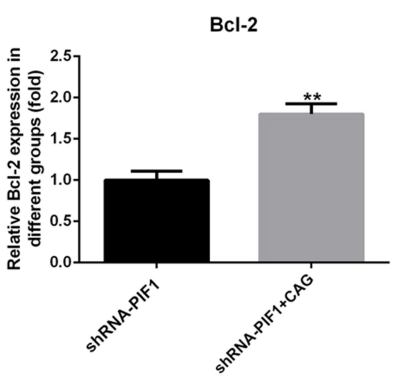

TERT

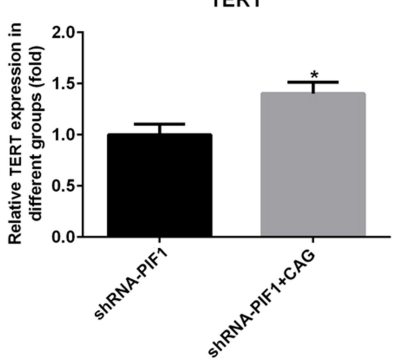

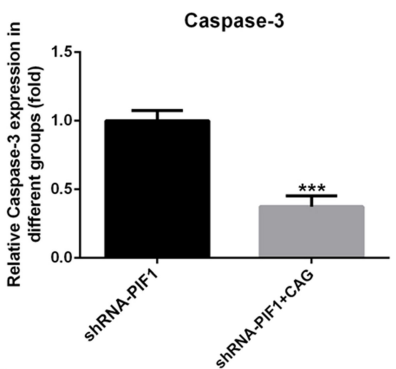

B

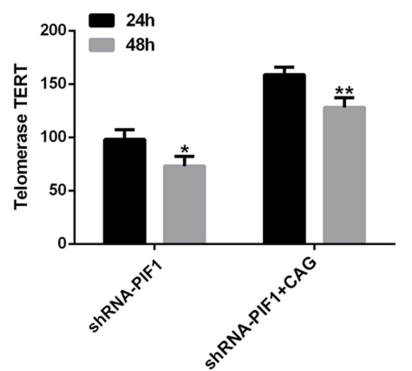

Figure 8 Cycloastragenol (CAG) affects the expressions of apoptosis-related proteins, PIFI and TERT. (A) The expression of Bax, Bcl-2 and Caspase-3 in C-33A cells after CAG treatment was analyzed by Western blot analysis. $* \mathrm{P}<0.05$, $* * \mathrm{P}<0.01$ and $* * * \mathrm{P}<0.001$ vs shRNA-PIFI group. (B) The expression of TERT in $\mathrm{C}-33 \mathrm{~A}$ cells after $\mathrm{CAG}$ treatment was analyzed by Western blot analysis. ${ }^{*} \mathrm{P}<0.05$ and ${ }^{*} * \mathrm{P}<0.01$ vs shRNA-PIFI group.

silencing could inhibit the growth of human cervical cancer cells, induce their apoptosis, and weaken their abilities of migration and invasion. Zhao et $\mathrm{al}^{19}$ indicated that miR491-5p could inhibit the expression of TERT and thus inhibit the proliferation of cervical cancer cells. PIF1 is a negative regulator of telomerase activity. ${ }^{20,21}$ As mentioned above, PIF1 helicase could inhibit TERT activity and dissociate telomeres, thus promoting cell apoptosis. Therefore, we wanted to investigate whether PIF1 could affect the proliferation and apoptosis by targeting TERT.
In this study, we found that PIF1 expression was increased in cervical cancer cells. PIF1 interference inhibited the proliferation, decreased the rate of $\mathrm{G} 2 / \mathrm{M}$ phase and promoted the apoptosis of C-33A cells which were reversed by PIF1 overexpression. PIF1 interference down-regulated the TERT expression and PIF1 overexpression upregulated the TERT expression.

CAG is the only telomerase activator found at present, which can delay the shortening of telomeres by increasing telomerase. $^{22}$ A study indicated that CAG could inhibit the 
apoptosis of rat neurons after cerebral ischemia-reperfusion by promoting the expression of TERT. ${ }^{23}$ In in vitro experiments, CAG could activate telomerase through the pathway of mitogen-activated protein kinase (MAPK). ${ }^{24}$ In in vivo experiments, as a telomerase active agent, CAG could increase TERT level of rats and participate in the repair of DNA damage. ${ }^{22}$ Cao et $\mathrm{al}^{25}$ found that CAG was a TERT activator of epidermal stem cells (EpSCs) and was associated with their proliferation and migration. In this study, CAG was used as TERT activator to demonstrate whether the effect of PIF1 interference on the proliferation and apoptosis of C-33A cells was based on TERT expression. The results indicated that $\mathrm{CAG}$ treatment could improve the proliferation, increase the rate of $\mathrm{G} 2 / \mathrm{M}$ phase and suppress the apoptosis of C-33A cells transfected with shRNA-PIF1.

In conclusion, PIF1 helicase could promote the proliferation, increase the rate of $\mathrm{G} 2 / \mathrm{M}$ phase and suppress the apoptosis of cervical cancer by up-regulating the expression of telomerase TERT. Our new finding may provide theoretical basis for drug discovery against cervical cancer.

\section{Acknowledgment}

This study is supported by the Zhejiang basic public welfare research program project fund (LQ20H270016).

\section{Disclosure}

The authors report no conflicts of interest for this work.

\section{References}

1. Small WA, Bacon M, Bajaj A, et al. Cervical cancer: a global health crisis. Cancer. 2017;123(13):2404-2412. doi:10.1002/cncr.30667

2. Cohen PA, Jhingran A, Oaknin A, Denny L. Cervical cancer. Lancet. 2019;393(10167):169-182. doi:10.1016/S0140-6736(18)32470-X

3. Lincz LF, Mudge L-M, Scorgie FE, Sakoff JA, Hamilton CS, Seldon M. Quantification of hTERT splice variants in melanoma by SYBR green real-time polymerase chain reaction indicates a negative regulatory role for the beta deletion variant. Neoplasia (New York, NY). 2008;10(10):1131-1137. doi:10.1593/neo.08644

4. Kyo S, Takakura M, Fujiwara T, Inoue M. Understanding and exploiting hTERT promoter regulation for diagnosis and treatment of human cancers. Cancer Sci. 2008;99(8):1528-1538. doi:10.1111/j.13497006.2008.00878.x

5. Cha N, Li X-Y, Zhao Y-J, Wang E-H, Wu G-P. hTERT gene amplification and clinical significance in pleural effusions of patients with lung cancer. Clin Lung Cancer. 2012;13(6):494-499. doi:10.1016/j. cllc.2012.01.007

6. Liu J-P, Nicholls C, Chen S-M, Li H, Tao -Z-Z. Strategies of treating cancer by cytokine regulation of chromosome end remodelling. Clin Exp Pharmacol Physiol. 2010;37(1):88-92. doi:10.1111/j.14401681.2009.05251.x

7. Visnovsky J, Kudela E, Farkasova A, Balharek T, Krkoska M, Danko J. Amplification of TERT and TERC genes in cervical intraepithelial neoplasia and cervical cancer. Neuro Endocrinol Lett. 2014;35(6):518-522.
8. Cassar L, Li H, Pinto AR, Nicholls C, Bayne S, Liu J-P. Bone morphogenetic protein-7 inhibits telomerase activity, telomere maintenance, and cervical tumor growth. Cancer Res. 2008;68 (22):9157-9166. doi:10.1158/0008-5472.CAN-08-1323

9. Zhang DH, Zhou B, Huang Y, Xu LX, Zhou JQ. The human Pif1 helicase, a potential Escherichia coli RecD homologue, inhibits telomerase activity. Nucleic Acids Res. 2006;34(5):1393-1404. doi:10.1093/nar/gk1029

10. Boulé J-B, Vega LR, Zakian VA. The yeast Piflp helicase removes telomerase from telomeric DNA. Nature. 2005;438(7064):57-61. doi:10.1038/nature04091

11. Gagou ME, Ganesh A, Phear G. Human PIF1 helicase supports DNA replication and cell growth under oncogenic-stress. Oncotarget. 2014;5(22):11381-11398. doi:10.18632/oncotarget.2501

12. Geronimo CL, Zakian VA. Getting it done at the ends: PIF1 family DNA helicases and telomeres. DNA Repair (Amst). 2016;44:151-158. doi:10.1016/j.dnarep.2016.05.021

13. Kirkpatrick KL, Mokbel K. The significance of human telomerase reverse transcriptase (hTERT) in cancer. Eur J Surg Oncol. 2001;27 (8):754-760. doi:10.1053/ejso.2001.1151

14. Wirth M, Russell-Eggitt I, Craig J, Elder J, Mackey D. Etiology of congenital and pediatric cataract in an Australian population. $\mathrm{Br}$ J Ophthalmol. 2002;86(7):782-786. doi:10.1136/bjo.86.7.782

15. Daniel M, Peek GW, Tollefsbol TO. Regulation of the human catalytic subunit of telomerase (hTERT). Gene. 2012;498(2):135-146. doi:10.1016/j.gene.2012.01.095

16. Zou M-X, Lv G-H, Li J, She X-L, Jiang Y. Upregulated human telomerase reverse transcriptase (hTERT) expression is associated with spinal chordoma growth, invasion and poor prognosis. $\mathrm{Am}$ J Transl Res. 2016;8(2):516-529.

17. Reddy MA, Francis PJ, Berry V, Bhattacharya SS, Moore AT. Molecular genetic basis of inherited cataract and associated phenotypes. Surv Ophthalmol. 2004;49(3):300-315. doi:10.1016/j. survophthal.2004.02.013

18. Zhu D, Ye M, Zhang W. E6/E7 oncoproteins of high risk HPV-16 upregulate MT1-MMP, MMP-2 and MMP-9 and promote the migration of cervical cancer cells. Int $J$ Clin Exp Pathol. 2015;8:4981-4989.

19. Zhao Q, Zhai Y-X, Liu H-Q, Shi Y-A, Li X-B. MicroRNA-491-5p suppresses cervical cancer cell growth by targeting hTERT. Oncol Rep. 2015;34(2):979-986. doi:10.3892/or.2015.4013

20. Lahaye A, Stahl H, Thines-Sempoux D, Foury F. PIF1: a DNA helicase in yeast mitochondria. EMBO J. 1991;10:997-1007. doi:10.1002/j.1460-2075.1991.tb08034.x

21. Lahaye A, Leterme S, Foury F. PIF1 DNA helicase from Saccharomyces cerevisiae. Biochemical characterization of the enzyme. J Biol Chem. 1994;268:26155-26161.

22. de Jesus BB, Schneeberger K, Vera E, Tejera A, Harley CB, Blasco MA. The telomerase activator TA-65 elongates short telomeres and increases health span of adult/old mice without increasing cancer incidence. Aging Cell. 2011;10(4):604-621. doi:10.1111/ j.1474-9726.2011.00700.x

23. Gao W. Effects of Cycloastragalus alcohol on neuronal apoptosis and TERT expression in cerebral ischemia-reperfusion rats. Chin J Pathophysiol. 2015;31(10):1858.

24. Yung L, Lam W, Ho M. Astragaloside IV and cycloastragenol stimulate the phosphorylation of extracellular signal-regulated protein kinase in multiple cell types. Planta Med. 2012;78(2):115-121. doi:10.1055/s-0031-1280346

25. Cao Y, Xu L, Yang X, et al. The potential role of cycloastragenol in promoting diabetic wound repair in vitro. Biomed Res Int. 2019;2019:7023950. 


\section{Publish your work in this journal}

Cancer Management and Research is an international, peer-reviewed open access journal focusing on cancer research and the optimal use of preventative and integrated treatment interventions to achieve improved outcomes, enhanced survival and quality of life for the cancer patient.
The manuscript management system is completely online and includes a very quick and fair peer-review system, which is all easy to use. Visit http://www.dovepress.com/testimonials.php to read real quotes from published authors.

Submit your manuscript here: https://www.dovepress.com/cancer-management-and-research-journal 